\title{
Distinct Characteristics of Escherichia coli Isolated from Patients with Urinary Tract Infections in a Medical Center at a Ten-Year Interval
}

\author{
Wei-Hung Lin ${ }^{1,2,+}$, Yen-Zhen Zhang ${ }^{3,+}$, Po-Yao Liu ${ }^{3,4,+}{ }^{+}$Po-Shun Chen ${ }^{5}$, Shining Wang ${ }^{3}$, Pei-Yun Kuo ${ }^{3}$, \\ Tran Thi Dieu Thuy ${ }^{3}$, Tran Thi Thuy Duong ${ }^{3}$, Li-Li Wen ${ }^{6}$, Yi-Hsien Hsieh ${ }^{7} \mathbb{D}$, Ming-Cheng Wang ${ }^{2,8, *}$ \\ and Cheng-Yen Kao ${ }^{3, *(\mathbb{D})}$
}

Citation: Lin, W.-H.; Zhang, Y.-Z.; Liu, P.-Y.; Chen, P.-S.; Wang, S.; Kuo, P.-Y.; Thuy, T.T.D.; Duong, T.T.T.; Wen, L.-L.; Hsieh, Y.-H.; et al. Distinct Characteristics of Escherichia coli Isolated from Patients with Urinary Tract Infections in a Medical Center at a Ten-Year Interval. Pathogens 2021, 10, 1156. https://doi.org/10.3390/ pathogens 10091156

Academic Editors: Gabriela Jorge Da Silva and Sara Domingues

Received: 8 August 2021

Accepted: 7 September 2021

Published: 8 September 2021

Publisher's Note: MDPI stays neutral with regard to jurisdictional claims in published maps and institutional affiliations.

Copyright: (c) 2021 by the authors. Licensee MDPI, Basel, Switzerland. This article is an open access article distributed under the terms and conditions of the Creative Commons Attribution (CC BY) license (https:// creativecommons.org/licenses/by/ $4.0 /)$.
1 Institute of Clinical Medicine, College of Medicine, National Cheng Kung University, Tainan 701, Taiwan; dindonwhlin@hotmail.com

2 Division of Nephrology, Department of Internal Medicine, National Cheng Kung University Hospital, College of Medicine, National Cheng Kung University, Tainan 701, Taiwan

3 Institute of Microbiology and Immunology, School of Life Sciences, National Yang Ming Chiao Tung University, Taipei 112, Taiwan; yenjean0326@gmail.com (Y.-Z.Z.); eric50215@gmail.com (P.-Y.L.); i34044025@gs.ncku.edu.tw (S.W.); togebi87303@gmail.com (P.-Y.K.); dieuthuy28021997@gmail.com (T.T.D.T.) tranthithuyduong190198@gmail.com (T.T.T.D.)

4 Department of Biotechnology and Laboratory Science in Medicine, School of Biomedical Science and Engineering, National Yang Ming Chiao Tung University, Taipei 112, Taiwan

5 Department of Biomedical Engineering, University of Southern California, Los Angeles, CA 90089-0482, USA; poshunch@usc.edu

6 Department of Clinical Laboratory, En Chu Kong Hospital, New Taipei City 237, Taiwan; 10036@km.eck.org.tw

7 Institute of Medicine, Chung Shan Medical University, Taichung 402, Taiwan; hyhsien@csmu.edu.tw

8 Institute of Clinical Pharmacy and Pharmaceutical Sciences, College of Medicine, National Cheng Kung University, Tainan 701, Taiwan

* Correspondence: wangmc@mail.ncku.edu.tw (M.-C.W.); kaocy@ym.edu.tw (C.-Y.K.); Tel.: +886-2-28267112 (C.-Y.K.); Fax: +886-2-28212880 (C.-Y.K.)

+ These authors contributed equally to this manuscript.

Abstract: Escherichia coli causing urinary tract infections (UTIs) are one of the most common outpatient bacterial infections. This study aimed to compare the characteristics of E. coli isolated from UTI patients in a single medical center in 2009-2010 $(n=504)$ and $2020(n=340)$. The antimicrobial susceptibility of $E$. coli was determined by the disk diffusion method. PCRs were conducted to detect phylogenetic groups, ST131, K1 capsule antigen, and 15 virulence factors. Phylogenetic group B2 dominated in our 2009-2010 and 2020 isolates. Moreover, no phylogenetic group E strains were isolated in 2020. E. coli isolates in 2020 were more susceptible to amoxicillin, ampicillin/sulbactam, cefuroxime, cefmetazole, ceftazidime, cefoxitin, tetracycline, and sulfamethoxazole/trimethoprim, compared to the isolates in 2009-2010. Extensively drug-resistant (XDR)-E. coli in 2009-2010 were detected in groups B1 (5 isolates), B2 (12 isolates), F (8 isolates), and unknown (1 isolate). In 2020, XDR-E. coli were only detected in groups A (2 isolates), B2 (5 isolates), D (1 isolate), and F (4 isolates). The prevalence of virulence factor genes aer and fimH were higher in E. coli in 2009-2010 compared to those in 2020. In contrast, afa and sat showed higher frequencies in E. coli isolates in 2020 compared to $E$. coli in 2009-2010.

Keywords: antimicrobial susceptibility; longitudinal surveillance; phylogenetic groups; urinary tract infections; virulence factors

\section{Introduction}

Urinary tract infection (UTI) is one of the most common outpatient bacterial infections worldwide with a lifetime incidence of $50-60 \%$ in adult women and contribute to a substantial financial burden on society [1]. Lower UTI refers to the inflammation and 
infection of the bladder and urethra [2]. In contrast, upper UTI severely affects the function of kidneys and could be potentially life-threatening when bacteria invade the bloodstream from infected kidneys to cause urosepsis [2]. UTI in young, healthy, non-pregnant, premenopausal female patients with anatomically and functionally normal urinary tract is called uncomplicated UTI. However, UTI associated with host risk factors such as host immunodeficiency, urinary tract abnormality, bladder dysfunction in type 2 diabetes, and estrogen deficiency, with increasing colonization and decreasing efficacy of therapy, is defined as complicated UTI [3,4].

The elderly are more susceptible to uropathogenic Escherichia coli (UPEC), which is the dominant infectious pathogen in both uncomplicated and complicated UTIs [1,5]. UPEC strains show great diversity in their gene content, virulence factors, genomic organization, and pathogenicity islands [6]. Several virulence factors of UPEC are shown to be related to the pathogenesis of bacterial UTI [1,7-10]. Wang et al. reported that regarding the bacterial characteristics in diabetic patients with UPEC, the isolated E. coli strains had more virulence factor genes, including $\mathrm{K} 1$ capsule neuA, adhesin pap GII, afimbrial adhesin afa, and hemolysin hlyA [11]. Type 1 fimbrial adhesin FimH has a critical role not only in lower UTI pathogenesis but also in kidney infections by acting synergistically with PapGII [8]. Moreover, fimbriae [12], iron acquisition systems [13], iron-regulated gene homologue adhesin Iha [14], ferric aerobactin receptor IutA [15], cytotoxic necrotizing factor 1 (Cnf1) [16], hemolysin (HlyA) [16], uropathogenic specific protein (Usp) [17], and outer membrane protease $\mathrm{T}(\mathrm{OmpT})$ [18], are also shown as important virulence factors of $E$. coli in murine uropathogenesis. Carriage of these urovirulence factors is thought to enhance UPEC pathogenicity and is used to measure and categorize clinical UPEC strains isolated from different patient populations [19-21]. Moreover, E. coli isolated from the elderly have fewer virulence factors compared to those isolated from younger age groups [5].

Antibiotic therapy is an effective approach to reduce the duration of UTI symptoms. First-line antibiotics such as trimethoprim and some $\beta$-lactams, or second-line quinolones and amoxicillin in combination with clavulanic acid, are considered effective treatments for UTIs. In addition, carbapenems were considered the last resort treatment for infections caused by extended-spectrum $\beta$-lactamases-producing Enterobacterales. In the last decade, the extensive use of antibiotics has resulted in the emergence of antibiotic-resistant pathogens and leads to the spread of antibiotic resistance [22]. Importantly, carbapenemresistant UPEC in community-acquired UTIs was also reported worldwide [23,24].

Although the association of host factors and bacterial virulence genes with the pathogenesis of E. coli causing UTIs has been reported $[5,25,26]$, the longitudinal survey to compare the characteristics of $E$. coli isolated from a single medical center to determine the distinct characteristics of UTI-causing E. coli at a 10-year interval is rare. In this study, we aimed to compare the bacterial characteristics, including phylogenetic groups, antibiotic susceptibility, and virulence factors, of E. coli strains isolated from UTI patients in 2009-2010 and 2020.

\section{Results}

\subsection{The Different Distribution of Phylogenetic Groups amongst E. coli Isolates at a 10-Year Interval}

We previously divided UTI patients into six age groups $(\leq 3,4-20,21-40,41-60,61-80$, and $>80$ years old) and reported that $E$. coli isolated from the elderly with UTI were more resistant to antimicrobial agents and had fewer virulence factors compared to $E$. coli isolated from the younger age groups [5]. To exclude the patient's age effect, we randomly selected 504 and 340 E. coli isolated from six patient age groups with UTI in 2009-2010 and 2020, respectively (Table 1). The average age of patients with E. coli causing UTI in 2009-2010 was 44.47 years, with $73.4 \%$ of these E. coli were isolated from women (Table 1 ). The average age of patients with E. coli causing UTI in 2020 was 47.6 years, and $65.3 \%$ of these E. coli were isolated from women (Table 1). 
Table 1. The distribution of the gender and average age of UTI patients in 2009-2010 and 2020.

\begin{tabular}{ccc}
\hline & \multicolumn{2}{c}{ Year of Isolation } \\
\cline { 2 - 3 } & $\mathbf{2 0 0 9 - 2 0 1 0}(\mathbf{n}=\mathbf{5 0 4})$ & $\mathbf{2 0 2 0}(\mathbf{n}=\mathbf{3 4 0})$ \\
\hline Average age (year) & 44.47 & 47.76 \\
\hline Age group (years old), n (\%) & $75(14.9)$ & $60(17.6)$ \\
\hline$\leq 3$ & $62(12.3)$ & $17(5.0)$ \\
$21-40$ & $97(19.2)$ & $62(18.2)$ \\
$41-60$ & $89(17.7)$ & $66(19.4)$ \\
$61-80$ & $92(18.3)$ & $68(20.0)$ \\
$>80$ & $89(17.7)$ & $67(19.7)$ \\
\hline Gender, $\mathrm{n}(\%)$ & & \\
\hline Female & $370(73.4)$ & $222(65.3)$ \\
Male & $134(26.6)$ & $118(34.7)$ \\
\hline
\end{tabular}

Phylogenetic analysis has classified E. coli strains into eight groups (A, B1, B2, C, D, E, F, and clade I) [27]. We next examined the distribution of phylogenetic groups among our 844 isolates. The results showed that the distribution of phylogenetic groups in $E$. coli was different in 2009-2010 and $2020(p<0.001)$. Phylogenetic group B2 dominated in our 2009-2010 isolates (313 isolates, 62.1\%), followed by group B1 (44 isolates, $8.7 \%$ ), group D (43 isolates, $8.5 \%$ ), group C (33 isolates, $6.5 \%$ ), group $\mathrm{F}$ ( 32 isolates, $6.3 \%$ ), group E (15 isolates, $3.0 \%$ ), unknown (14 isolates, $2.8 \%$ ), group A (9 isolates, $1.8 \%$ ), and clade I (1 isolate, $0.2 \%)$. Phylogenetic group B2 was also dominated in our 2020 isolates (234 isolates, $68.8 \%$ ), followed by group D (29 isolates, $8.5 \%$ ), group B1 (22 isolates, $6.5 \%$ ), group F (19 isolates, 5.6\%), group A (17 isolates, 5.0\%), unknown (10 isolates, $2.9 \%$ ), group C (6 isolates, $1.8 \%)$, and clade I ( 3 isolates, $0.9 \%)$. Interestingly, no phylogenetic group $\mathrm{E}$ was isolated in 2020. In summary, the predominant phylogenetic group was B2 $(547 / 844,64.8 \%)$, followed by D (72/844, 8.5\%), B1 (66/844, 7.8\%), F $(51 / 844,6.0 \%), \mathrm{C}$ $(38 / 844,4.6 \%)$, A (26/844, 3.1\%), unknown $(24 / 844,2.8 \%)$, and clade I $(4 / 8440.5 \%)$. E. coli sequence type 131 (ST131) has emerged rapidly to become the most drug-resistant and prevalent extraintestinal pathogenic E. coli clone in circulation worldwide. Therefore, we next determined the prevalence of ST131 in our 2009-2010 and 2020 isolates by PCR with ST131 specific primers. The results showed that $69(13.7 \%)$ and $33(9.7 \%)$ of $2009-2010$ and 2020 E. coli were ST131, respectively $(p=0.082)$.

\subsection{Antimicrobial Susceptibility of E. coli Isolated from UTI Patients}

We further compared the antimicrobial susceptibility (21 antimicrobials divided into 11 categories) of E. coli isolates collected in 2009-2010 and 2020. We found all UTI isolates were susceptible to tigecycline. Interestingly, E. coli isolates in 2020 were more susceptible to amoxicillin $(p=0.011)$, ampicillin/sulbactam $(p=0.001)$, cefuroxime $(p=0.018)$, cefmetazole $(p<0.001)$, ceftazidime $(p<0.001)$, cefoxitin $(p<0.001)$, tetracycline $(p=0.011)$, and sulfamethoxazole/trimethoprim ( $p=0.010$ ), compared to the isolates in 2009-2010 (Table 2). In general, our UTI isolates were highly susceptible ( $>90 \%$ susceptibility) to amikacin, piperacillin/tazobactam, imipenem, ertapenem, meropenem, but were resistant $(<60 \%$ susceptibility) to ampicillin, ciprofloxacin, tetracycline, and sulfamethoxazole/trimethoprim (Table 2). 
Table 2. Antimicrobial susceptibility of UTI E. coli isolates in 2009-2010 and 2020.

\begin{tabular}{|c|c|c|c|c|c|c|c|}
\hline & \multicolumn{6}{|c|}{ Year of Isolation } & \multirow[b]{2}{*}{$p$-Value } \\
\hline & \multicolumn{3}{|c|}{$2009-2010(n=504)$} & \multicolumn{3}{|c|}{$2020(n=340)$} & \\
\hline \multicolumn{8}{|c|}{ Antimicrobial Category and Agents } \\
\hline Aminoglycoside & S & I & $\mathrm{R}$ & S & I & $\mathrm{R}$ & \\
\hline AN & $486(98.4)$ & $2(0.4)$ & $6(1.2)$ & 337 (99.1) & $1(0.3)$ & $2(0.6)$ & 0.376 \\
\hline GM & $360(71.4)$ & $14(2.8)$ & $130(25.8)$ & $263(77.4)$ & $7(2.1)$ & $70(20.6)$ & 0.055 \\
\hline \multicolumn{8}{|l|}{ Penicillins } \\
\hline AM & $115(22.8)$ & $1(0.2)$ & $388(77.0)$ & $89(26.2)$ & $6(1.8)$ & $245(72.1)$ & 0.264 \\
\hline AMC & $329(65.3)$ & $44(8.7)$ & $131(26)$ & $250(73.5)$ & $44(12.9)$ & $46(13.5)$ & 0.011 \\
\hline \multicolumn{8}{|c|}{ Penicillins $+\beta$-lactamase inhibitors } \\
\hline SAM & $335(66.5)$ & $62(12.3)$ & $107(21.2)$ & $265(77.9)$ & $24(7.1)$ & $51(15.0)$ & $<0.001$ \\
\hline $\mathrm{TZP}$ & $483(95.8)$ & $16(3.2)$ & $5(1.0)$ & $331(97.4)$ & $6(1.8)$ & $3(0.9)$ & 0.242 \\
\hline \multicolumn{8}{|l|}{ Carbapenems } \\
\hline IPM & $501(99.4)$ & $3(0.6)$ & $0(0)$ & $336(98.8)$ & $2(0.6)$ & $2(0.6)$ & 0.361 \\
\hline ETP & 497 (98.6) & $3(0.6)$ & $4(0.8)$ & $338(99.4)$ & $1(0.3)$ & $1(0.3)$ & 0.267 \\
\hline MEM & $502(99.6)$ & $0(0)$ & $2(0.4)$ & $338(99.4)$ & $0(0)$ & $2(0.6)$ & 0.691 \\
\hline \multicolumn{8}{|c|}{$\begin{array}{l}\text { Non-extended spectrum } \\
\text { cephalosporins }\end{array}$} \\
\hline $\mathrm{CZ}$ & $338(67.1)$ & $0(0)$ & $166(32.9)$ & $248(72.9)$ & $0(0)$ & $92(27.1)$ & 0.069 \\
\hline CXM & $340(67.5)$ & $35(6.9)$ & $129(25.6)$ & $255(75.0)$ & $7(2.1)$ & $78(22.9)$ & 0.018 \\
\hline $\mathrm{CMZ}$ & $422(83.7)$ & $29(5.8)$ & $53(10.5)$ & $323(95.0)$ & $1(0.3)$ & $16(4.7)$ & $<0.001$ \\
\hline \multicolumn{8}{|l|}{$\begin{array}{l}\text { Extended-spectrum } \\
\text { cephalosporins }\end{array}$} \\
\hline CRO & $343(68.1)$ & $18(3.6)$ & $143(28.4)$ & $252(74.1)$ & $4(1.2)$ & $84(24.7)$ & 0.058 \\
\hline CAZ & $364(72.2)$ & $31(6.2)$ & 109 (21.6) & $281(82.6)$ & $25(7.4)$ & $34(10.0)$ & $<0.001$ \\
\hline FEP & $432(85.7)$ & $29(5.8)$ & $43(8.5)$ & $289(85.0)$ & $16(4.7)$ & $35(10.3)$ & 0.773 \\
\hline \multicolumn{8}{|l|}{ Cephamycins } \\
\hline FOX & $380(75.4)$ & $19(3.8)$ & $105(20.8)$ & $310(91.2)$ & $6(1.8)$ & $24(7.1)$ & $<0.001$ \\
\hline \multicolumn{8}{|l|}{ Fluoroquinolones } \\
\hline CIP & $293(58.1)$ & $36(7.1)$ & $175(34.7)$ & $192(56.5)$ & $27(7.9)$ & $121(35.6)$ & 0.631 \\
\hline LVX & $335(66.5)$ & $8(1.6)$ & $161(31.9)$ & $223(65.6)$ & $5(1.5)$ & $112(32.9)$ & 0.791 \\
\hline \multicolumn{8}{|l|}{ Tetracyclines } \\
\hline TE & $210(41.7)$ & $23(4.6)$ & $271(53.8)$ & $172(50.6)$ & $10(2.9)$ & $158(46.5)$ & 0.011 \\
\hline \multicolumn{8}{|l|}{ Glycylcyclines } \\
\hline TIG & $504(100)$ & $0(0)$ & $0(0)$ & $340(100)$ & $0(0)$ & $0(0)$ & - \\
\hline \multicolumn{8}{|c|}{ Folate pathway inhibitors } \\
\hline SXT & $242(48.0)$ & $1(0.2)$ & $261(51.8)$ & $194(57.1)$ & $0(0)$ & $146(42.9)$ & 0.010 \\
\hline
\end{tabular}

Abbreviations: AM, ampicillin; AMC, amoxicillin; AN, amikacin; CAZ ceftazidime, CIP, ciprofloxacin; CMZ, cefmetazole; CRO, ceftriaxone; CXM, cefuroxime; CZ, cefazolin; ETP, ertapenem; FEP, cefepime; FOX, cefoxitin; GM, gentamicin; LVX, levofloxacin; IPM, imipenem; MEM meropenem; SAM, ampicillin/sulbactam; SXT, sulfamethoxazole/trimethoprim; TE, tetracycline; TIG, tigecycline; TZP, piperacillin/tazobactam; S, susceptible; I, intermediate resistant; R, resistant.

We next determined whether the antimicrobial susceptibility of E. coli isolates was associated with phylogenetic groups (Table 3). In this study, we determined the antimicrobial susceptibility of $E$. coli isolates to 21 antimicrobials (divided into 11 categories), and then we classified our isolates into multidrug-resistant (MDR, non-susceptible to $\geq$ one agent in $\geq$ three antimicrobial categories), extensively drug-resistant (XDR, non-susceptible to $\geq$ one agent in all but $\leq$ two categories), and pandrug-resistant (PDR, non-susceptible to all antimicrobial agents), according to the previous study [28]. We found 279 MDR-E. coli $(279 / 504,55.4 \%)$ and 26 XDR-E. coli $(26 / 504,5.2 \%)$ in the 2009-2010 collection, and 187 MDR-E. coli $(187 / 340,55.0 \%)$ and 12 XDR-E. coli $(12 / 340,3.5 \%)$ in the 2020 collection (Table 3). No PDR-E. coli was identified in our 844 isolates. Moreover, there was no significant difference in the prevalence of MDR- and XDR-E. coli in 2009-2010 compared to 2020 $(p=0.496)$. 
Table 3. The distribution of multidrug-resistant and extensively drug-resistant E. coli in 2009-2010 and 2020.

\begin{tabular}{|c|c|c|c|c|c|c|}
\hline \multirow[b]{3}{*}{ Drug-Resistant Isolate, n (\%) } & \multicolumn{6}{|c|}{ Year of Isolation } \\
\hline & \multicolumn{3}{|c|}{$2009-2010(n=504)$} & \multicolumn{3}{|c|}{$2020(n=340)$} \\
\hline & Non-MDR or XDR & MDR & XDR & Non-MDR or XDR & MDR & XDR \\
\hline A & $3(33.3)$ & $6(66.7)$ & $0(0)$ & $3(17.6)$ & $12(70.6)$ & $2(11.8)$ \\
\hline B1 & $7(15.9)$ & $32(72.7)$ & $5(11.4)$ & $12(54.5)$ & $10(45.5)$ & $0(0)$ \\
\hline B2 & $161(51.4)$ & $140(44.7)$ & $12(3.9)$ & $111(47.4)$ & $118(50.4)$ & $5(2.2)$ \\
\hline $\mathrm{C}$ & $4(12.1)$ & $29(87.9)$ & $0(0)$ & $1(16.7)$ & $5(83.3)$ & $0(0)$ \\
\hline $\mathrm{D}$ & $9(20.9)$ & $34(79.1)$ & $0(0)$ & $11(37.9)$ & $17(58.6)$ & $1(3.5)$ \\
\hline $\mathrm{E}$ & $3(20.0)$ & $12(80.0)$ & $0(0)$ & $0(0)$ & $0(0)$ & $0(0)$ \\
\hline $\mathrm{F}$ & $6(18.8)$ & $18(56.3)$ & $8(24.9)$ & $1(5.3)$ & $14(73.7)$ & $4(21.0)$ \\
\hline Clade I & $1(100)$ & $0(0)$ & $0(0)$ & $0(0)$ & $3(100)$ & $0(0)$ \\
\hline Unknown & $5(35.7)$ & $8(57.1)$ & $1(7.2)$ & $2(20.0)$ & $8(80.0)$ & $0(0)$ \\
\hline Total & $199(39.4)$ & $279(55.4)$ & $26(5.2)$ & $141(41.5)$ & $187(55.0)$ & $12(3.5)$ \\
\hline
\end{tabular}

Abbreviations: MDR, multidrug-resistant; XDR, extensively drug-resistant.

We further determined the distribution of MDR- (187/340, 55.0\%) and XDR-E. coli among different phylogenetic groups in 2009-2010 and 2020 (Table 3). The results showed a significant difference in the distribution of MDR- and XDR-E. coli with phylogenetic groups between the 2009-2010 and 2020 collections ( $p<0.001)$. In 2009-2010, 26 XDR-E. coli were identified in phylogenetic groups B1 (five isolates), B2 (12 isolates), F (eight isolates), and unknown (one isolate). In contrast, 12 XDR-E. coli were only identified in phylogenetic groups A (two isolates), B2 (five isolates), $\mathrm{D}$ (one isolate), and $\mathrm{F}$ (four isolates), in 2020 (Table 3). In addition, $87.9 \%, 80.0 \%$, and $79.1 \%$, of phylogenetic groups $C$, E, and D isolates, were MDR-E. coli, in 2009-2010 (Table 3). In contrast, $100 \%, 83.3 \%$, and $80.0 \%$, of phylogenetic groups clade I, C, and F isolates, were MDR-E. coli, in 2020 (Table 3).

\subsection{Antimicrobial Susceptibility of E. coli Isolated from UTI Patients Is Associated with Patient Age}

We previously reported that $E$. coli strains isolated from patient age group $>80$ were more resistant to amoxicillin in combined with clavulanate, cefazolin, cefixime, cefmetazole, ceftriaxone, cefuroxime, ciprofloxacin, levofloxacin, than the average resistant rate [5] In contrast, E. coli strains isolated from patient age group $\leq 3$ were more susceptible to amoxicillin in combined with clavulanate, cefixime, cefuroxime, ciprofloxacin, levofloxacin, and piperacillin/tazobactam, than the average resistant rate [5]. The distribution of antimicrobial resistance of $E$. coli isolated from different host age groups was shown in Table S1. Overall, E. coli isolated from the elderly were more resistant to cefazolin, cefuroxime, ceftriaxone, ciprofloxacin, and levofloxacin, in 2009-2010 and $2020(p<0.05)$ (Table S1). We next determined the antimicrobial susceptibility of $E$. coli isolated from two extreme age groups of UTI patients in 2009-2010 and 2020 (Table 4).

In 2009-2010 and 2020, E. coli strains isolated from patient age group $>80$ were more resistant to cefazolin, cefuroxime, ceftriaxone, ciprofloxacin, and levofloxacin $(p<0.05)$, than the E. coli isolated from the patient age group $\leq 3$ (Table 4). In 2009-2010, E. coli strains isolated from patient age group $>80$ were more resistant to amoxicillin $(p=0.007)$, ampicillin/sulbactam $(p=0.001)$, cefmetazole $(p<0.001)$, and ceftazidime $(p=0.006)$ (Table 4). Interestingly, E. coli strains isolated from patient age group $\leq 3$ were more resistant to sulfamethoxazole/trimethoprim ( $53.3 \%$ vs $35.8 \%, p=0.047)$, than the E. coli strains isolated from age group $>80$, in 2020 (Table 4). The distribution of MDR- and XDR-E. coli among these two patient age groups was not significantly different, in both 2009-2010 and 2020. Moreover, we found isolates in age group $>80$ in 2020 were more susceptible to ampicillin/sulbactam $(p=0.048)$, cefmetazole $(p=0.001)$, cefoxitin $(p=0.004)$, and sulfamethoxazole/trimethoprim $(p=0.002)$, compared to isolates in age group $>80$ in 2009-2010 (Table 4). 
Table 4. The distribution of antimicrobial-resistant E. coli in the age groups $\leq 3$ and $>80$ in 2009-2010 and 2020.

\begin{tabular}{|c|c|c|c|c|c|c|c|c|}
\hline \multirow{3}{*}{$\begin{array}{c}\text { Antimicrobial Category and } \\
\text { Agents }\end{array}$} & \multicolumn{3}{|c|}{$\begin{array}{l}\text { Age Group (Years Old) } \\
\text { in 2009-2010 }\end{array}$} & \multicolumn{3}{|c|}{$\begin{array}{l}\text { Age Group (Years Old) } \\
\text { in } 2020\end{array}$} & \multicolumn{2}{|c|}{$p$-Value } \\
\hline & $\begin{array}{c}\leq 3 \\
(n=75)\end{array}$ & $>80(\mathrm{n}=89)$ & $p$-Value & $\begin{array}{c}\leq 3 \\
(\mathrm{n}=60)\end{array}$ & $>80(n=67)$ & $p$-Value & $\begin{array}{l}\leq 3 \text { Age } \\
\text { Group }{ }^{a}\end{array}$ & $\begin{array}{l}>80 \text { Age } \\
\text { Group b }\end{array}$ \\
\hline & $\mathbf{I}+\mathbf{R}$ & $\mathbf{I}+\mathbf{R}$ & & $\mathbf{I}+\mathbf{R}$ & $\mathbf{I}+\mathbf{R}$ & & & \\
\hline \multicolumn{9}{|l|}{ Aminoglycoside } \\
\hline AN & $1(1.3)$ & $2(2.2)$ & 0.664 & $0(0)$ & $1(1.5)$ & 0.342 & 0.369 & 0.734 \\
\hline GM & $25(33.3)$ & $25(28.1)$ & 0.467 & $17(28.3)$ & $14(20.9)$ & 0.330 & 0.533 & 0.304 \\
\hline \multicolumn{9}{|l|}{ Penicillins } \\
\hline $\mathrm{AM}$ & $62(82.7)$ & $74(83.1)$ & 0.935 & $44(73.3)$ & $51(76.1)$ & 0.718 & 0.189 & 0.276 \\
\hline AMC & $17(22.7)$ & $38(42.7)$ & 0.007 & $16(26.7)$ & $22(32.8)$ & 0.448 & 0.591 & 0.210 \\
\hline \multicolumn{9}{|l|}{ Penicillins $+\beta$-lactamase inhibitors } \\
\hline SAM & $14(18.7)$ & $39(43.8)$ & 0.001 & $12(20.0)$ & $19(28.4)$ & 0.274 & 0.845 & 0.048 \\
\hline TZP & $1(1.3)$ & $6(6.7)$ & 0.088 & $0(0)$ & $2(3.0)$ & 0.177 & 0.369 & 0.292 \\
\hline \multicolumn{9}{|l|}{ Carbapenems } \\
\hline IPM & $0(0)$ & $0(0)$ & - & $0(0)$ & $2(3.0)$ & 0.177 & - & 0.101 \\
\hline ETP & $1(1.3)$ & $2(2.2)$ & 0.664 & $0(0)$ & $0(0)$ & - & 0.369 & 0.217 \\
\hline MEM & $1(1.3)$ & $1(1.1)$ & 0.903 & $0(0)$ & $1(1.5)$ & 0.342 & 0.369 & 0.839 \\
\hline \multicolumn{9}{|l|}{$\begin{array}{l}\text { Non-extended spectrum } \\
\text { cephalosporins }\end{array}$} \\
\hline $\mathrm{CZ}$ & $15(20.0)$ & $39(43.8)$ & 0.001 & $15(25.0)$ & $31(46.3)$ & 0.013 & 0.487 & 0.761 \\
\hline CXM & $15(20.0)$ & 37 (41.6) & 0.003 & $14(23.3)$ & $28(41.8)$ & 0.027 & 0.639 & 0.978 \\
\hline $\mathrm{CMZ}$ & $2(2.7)$ & $22(24.7)$ & $<0.001$ & $1(1.7)$ & $6(9.0)$ & 0.072 & 0.695 & 0.011 \\
\hline \multicolumn{9}{|l|}{$\begin{array}{l}\text { Extended-spectrum } \\
\text { cephalosporins }\end{array}$} \\
\hline CRO & $16(21.3)$ & 37 (41.6) & 0.006 & $14(23.3)$ & $31(46.3)$ & 0.007 & 0.781 & 0.558 \\
\hline CAZ & $12(16.0)$ & $31(34.8)$ & 0.006 & $10(16.7)$ & $16(23.9)$ & 0.314 & 0.917 & 0.140 \\
\hline FEP & $9(12.0)$ & $19(21.3)$ & 0.113 & $10(16.7)$ & $15(22.4)$ & 0.418 & 0.438 & 0.876 \\
\hline \multicolumn{9}{|l|}{ Cephamycins } \\
\hline FOX & $8(10.7)$ & $30(33.7)$ & $<0.001$ & $3(4.9)$ & $9(13.4)$ & 0.105 & 0.232 & 0.004 \\
\hline \multicolumn{9}{|l|}{ Fluoroquinolones } \\
\hline CIP & $20(26.7)$ & $51(57.3)$ & $<0.001$ & $20(33.3)$ & $39(58.2)$ & 0.005 & 0.399 & 0.910 \\
\hline LVX & $15(20.0)$ & $43(48.3)$ & $<0.001$ & $16(26.7)$ & $36(53.7)$ & 0.002 & 0.360 & 0.503 \\
\hline \multicolumn{9}{|l|}{ Tetracyclines } \\
\hline TIG & $0(0)$ & $0(0)$ & _- & $0(0)$ & $0(0)$ & - & - & _- \\
\hline \multicolumn{9}{|l|}{ Folate pathway inhibitors } \\
\hline SXT & $41(54.7)$ & $54(60.7)$ & 0.438 & $32(53.3)$ & $24(35.8)$ & 0.047 & 0.877 & 0.002 \\
\hline Drug-resistance & & & 0.052 & & & 0.145 & 0.467 & 0.855 \\
\hline MDR & $39(52.0)$ & $57(64.0)$ & & $36(60.0)$ & $40(59.7)$ & & & \\
\hline XDR & $1(1.3)$ & $5(5.6)$ & & $0(0)$ & $4(6.0)$ & & & \\
\hline
\end{tabular}

${ }^{a}$ Statistical results for the distribution of virulence factors in patient age group $\leq 3$ years old in 2009-2010 compared to 2020. ${ }^{\mathrm{b}}$ Statistical results for the distribution of virulence factors in patient age group $>80$ years old in 2009-2010 compared to 2020. Abbreviations: AM, ampicillin; AMC, amoxicillin; AN, amikacin; CAZ ceftazidime, CIP, ciprofloxacin; CMZ, cefmetazole; CRO, ceftriaxone; CXM, cefuroxime; CZ, cefazolin; ETP, ertapenem; FEP, cefepime; FOX, cefoxitin; GM, gentamicin; LVX, levofloxacin; IPM, imipenem; MEM meropenem; SAM, ampicillin/sulbactam; SXT, sulfamethoxazole/trimethoprim; TE, tetracycline; TIG, tigecycline; TZP, piperacillin/tazobactam; I, intermediate resistant; $R$, resistant; MDR, multidrug-resistant; XDR, extensively drug-resistant.

2.4. The Different Prevalence of Bacterial Virulence Factors in E. coli Isolated from UTI Patients at a 10-Year Interval

To characterize the E. coli isolated from UTI patients in 2009-2010 and 2020, we determined the presence of $\mathrm{K} 1$ capsule antigen and 15 virulence factors in 844 isolates by PCR (Table 5). Overall, virulence factor genes aer, usp, ompT, and fimH, were found in more than $60 \%$ of 844 isolates (Table 5). The results showed that the prevalence of virulence factor genes aer $(64.3 \%$ vs $55.6, p=0.011)$ and fimH $(97.2 \%$ vs $89.1 \%, p<0.001)$, were higher in E. coli in 2009-2010, compared to those in 2020 (Table 5). In contrast, afa (63.8\% vs $56.3 \%$, $p=0.030)$ and sat $(40.9 \%$ vs $32.7 \%, p=0.016)$, showed higher frequencies in E. coli in 2020, compared to E. coli in 2009-2010 (Table 5). 
Table 5. The distribution of virulence factor genes in UTI E. coli in 2009-2010 and 2020.

\begin{tabular}{ccccc}
\hline $\begin{array}{c}\text { Virulence Factor } \\
\text { Genes }\end{array}$ & $\begin{array}{c}\mathbf{2 0 0 9 - 2 0 1 0} \\
\mathbf{( n = 5 0 4 )}\end{array}$ & $\begin{array}{c}\mathbf{2 0 2 0} \\
\mathbf{( n = 3 4 0 )}\end{array}$ & $\begin{array}{c}\text { Total } \\
\mathbf{( n = 8 4 4 )}\end{array}$ & $p$-Value \\
\hline papGI & $0(0)$ & $1(0.3)$ & $1(0.1)$ & 0.223 \\
papGII & $144(28.6)$ & $77(22.6)$ & $221(26.2)$ & 0.055 \\
papGIII & $85(16.9)$ & $41(12.1)$ & $126(14.9)$ & 0.055 \\
sfa & $43(8.5)$ & $43(12.6)$ & $86(10.2)$ & 0.053 \\
foc & $53(10.5)$ & $40(11.8)$ & $93(11.0)$ & 0.570 \\
cnf1 & $112(22.2)$ & $92(27.1)$ & $204(24.2)$ & 0.107 \\
aer & $324(64.3)$ & $189(55.6)$ & $513(60.8)$ & 0.011 \\
usp & $318(63.1)$ & $200(58.8)$ & $518(61.4)$ & 0.211 \\
iha & $180(35.7)$ & $111(32.6)$ & $291(34.5)$ & 0.358 \\
ompT & $400(79.4)$ & $253(74.4)$ & $653(77.4)$ & 0.092 \\
afa & $284(56.3)$ & $217(63.8)$ & $501(59.4)$ & 0.030 \\
iRONE & $20(40.3)$ & $121(35.6)$ & $324(38.4)$ & 0.169 \\
fimH & $490(97.2)$ & $303(89.1)$ & $793(94.0)$ & $<0.001$ \\
hly & $125(24.8)$ & $105(30.9)$ & $230(27.3)$ & 0.052 \\
sat & $165(32.7)$ & $139(40.9)$ & $304(36.0)$ & 0.016 \\
K1 & $126(25.0)$ & $75(22.1)$ & $201(23.8)$ & 0.325 \\
\hline
\end{tabular}

The distribution of virulence facctors in E. coli isolated from different host age groups was shown in Table S2. The prevalence of papGII, cnfI, usp, and ompT, were decreased in E. coli isolated from the elderly in both 2009-2010 and $2020(p<0.05)$ (Table S2). When the strains isolated from patient age groups $\leq 3$ and $>80$ were compared, we found strains isolated from the elderly age group had fewer virulence factors, including pap GII $(p=0.001)$, papGIII ( $p=0.028)$, cnf1 ( $p=0.015), u s p(p=0.009)$, and hlyA $(p=0.001)$, compared to the E. coli isolated from patient age group $\leq 3$, in 2009-2010 (Table 6). In 2020, the prevalence of virulence factors such as papGII $(p<0.001)$, foc $(p<0.038)$, cnf1 $(p=0.005)$, and aer $(p=0.024)$, were lower in the patient age group $>80$ compared to $\leq 3$ age group (Table 6). Moreover, we found isolates in age group $\leq 3$ in 2020 have less papGIII $(6.7 \%$ vs $22.7 \%, p=0.011)$ but more $a f a(75.0 \%$ vs $52.0 \%, p=0.006)$, compared to isolates in age group $\leq 3$ in $2009-2010$ (Table 6).

Table 6. The distribution of virulence factors in E. coli in $\leq 3$ and $>80$ age groups in 2009-2010 and 2020.

\begin{tabular}{|c|c|c|c|c|c|c|c|c|}
\hline \multirow[b]{2}{*}{$\begin{array}{l}\text { Virulence Factor } \\
\text { Genes }^{\text {a }}\end{array}$} & \multicolumn{3}{|c|}{$\begin{array}{c}\text { Age Group (Years Old) } \\
\text { in 2009-2010 }\end{array}$} & \multicolumn{3}{|c|}{$\begin{array}{c}\text { Age Group (Years Old) } \\
\text { in } 2020\end{array}$} & \multicolumn{2}{|c|}{$p$-Value } \\
\hline & $\begin{aligned} & \leq 3 \\
(\mathrm{n} & =75)\end{aligned}$ & $\begin{array}{c}>80 \\
(n=89)\end{array}$ & $p$-Value & $\begin{array}{c}\leq 3 \\
(n=60)\end{array}$ & $\begin{array}{c}>80 \\
(n=67)\end{array}$ & $p$-Value & $\begin{array}{l}\leq 3 \text { Age } \\
\text { Group }\end{array}$ & $\begin{array}{l}>80 \text { Age } \\
\text { Group } b\end{array}$ \\
\hline papGI & $0(0)$ & $0(0)$ & - & $0(0)$ & $0(0)$ & - & - & - \\
\hline papGII & $35(46.7)$ & $19(21.3)$ & 0.001 & $30(50.0)$ & $10(14.9)$ & $<0.001$ & 0.700 & 0.307 \\
\hline papGIII & $17(22.7)$ & $9(10.1)$ & 0.028 & $4(6.7)$ & $8(11.9)$ & 0.310 & 0.011 & 0.717 \\
\hline$s f a$ & $10(13.3)$ & $9(10.1)$ & 0.521 & $9(15.0)$ & 8 (11.9) & 0.613 & 0.782 & 0.717 \\
\hline foc & $8(10.7)$ & $6(6.7)$ & 0.370 & $12(20.0)$ & $5(7.5)$ & 0.038 & 0.129 & 0.862 \\
\hline cnf1 & $26(34.7)$ & $16(18.0)$ & 0.015 & $23(38.3)$ & $11(16.4)$ & 0.005 & 0.660 & 0.799 \\
\hline aer & $56(74.7)$ & $62(69.7)$ & 0.477 & $47(78.3)$ & $40(59.7)$ & 0.024 & 0.619 & 0.195 \\
\hline$u s p$ & $56(74.7)$ & 49 (55.1) & 0.009 & $43(71.7)$ & 39 (58.2) & 0.113 & 0.695 & 0.694 \\
\hline iha & 37 (49.3) & $36(40.4)$ & 0.254 & $24(40.0)$ & $27(40.3)$ & 0.973 & 0.279 & 0.985 \\
\hline ompT & $65(86.7)$ & $68(76.4)$ & 0.094 & $50(83.3)$ & $54(80.6)$ & 0.689 & 0.588 & 0.530 \\
\hline$a f a$ & 39 (52.0) & $50(56.2)$ & 0.592 & $45(75.0)$ & 40 (59.7) & 0.067 & 0.006 & 0.659 \\
\hline iRONE & 31 (41.3) & 29 (32.6) & 0.247 & $24(40.0)$ & $19(28.4)$ & 0.166 & 0.876 & 0.571 \\
\hline fimH & 74 (98.7) & $84(94.4)$ & 0.145 & 56 (93.3) & $61(91.0)$ & 0.633 & 0.103 & 0.420 \\
\hline hly & $33(44.0)$ & $17(19.1)$ & 0.001 & $25(41.7)$ & $18(26.9)$ & 0.078 & 0.786 & 0.250 \\
\hline sat & $36(48.0)$ & $34(38.2)$ & 0.206 & $28(46.7)$ & $29(43.3)$ & 0.702 & 0.877 & 0.522 \\
\hline K1 & $18(24.0)$ & $17(19.1)$ & 0.446 & $11(18.3)$ & $17(25.4)$ & 0.339 & 0.426 & 0.348 \\
\hline
\end{tabular}

a Statistical results for the distribution of virulence factors in patient age group $\leq 3$ years old in 2009-2010 compared to 2020. ${ }^{b}$ Statistical results for the distribution of virulence factors in patient age group $>80$ years old in 2009-2010 compared to 2020. 


\section{Discussion}

The longitudinal survey to investigate the distinct characteristics of $E$. coli isolated from a single medical center at a 10-year interval is rare. In this study, we compared the bacterial characteristics, including phylogenetic groups, antimicrobial susceptibility, and virulence factors, of E. coli strains isolated from patients with UTI in 2009-2010 and 2020. We applied modified phylogenetic analysis to classify E. coli strains into eight groups in this study. Although phylogenetic group B2 was predominant in our 2009-2010 (62.1\%) and $2020(68.8 \%)$ E. coli isolates, we found $38(4.6 \%)$ and $51(6.0 \%)$ isolates in our 844 isolates belonged to phylogenetic groups $C$ and E, respectively. Moreover, the results showed a decrease of groups $C$ and $\mathrm{E}$ at a 10-year interval. Iranpour et al. reported the predominant phylogenetic group was B2 (39.3\%), followed by unknown $(27.1 \%), \mathrm{E}(9.3 \%), \mathrm{C}$ and clade I (each 6.4\%), B1 (5\%), F and D (each 2.9\%), and A (0.7\%) in E. coli causing UTI in Iran [29]. These results suggest the geographical difference of characteristics of $E$. coli isolated from UTI patients in different countries. Our previous study showed that phylogenetic group B2 dominated in UTI isolates $(541 / 907,59.6 \%)$, followed by group D $(188 / 907,20.7 \%)$, group A $(95 / 907,10.5 \%)$, and group B1 $(83 / 907,10.5)$, according to old phylogenetic analysis method [30]. Compared to our previous report [30], we found a significant decrease of group D (20.7\% vs. $8.5 \%)$ and group A (10.5\% and $3.1 \%)$ isolates in this study. Gordon et al. reported that $21 \%$ of $E$. coli strains were either incorrectly assigned to a phylogenetic group by the old triplex method or could not be assigned to one of the phylogenetic groups $\mathrm{A}$, B1, B2, D, or E using the MLST data [31]. Therefore, this inconsistency may result from the incorrect assignment of $E$. coli isolates to a phylogenetic group, and thus the application of modified phylogenetic analysis to precisely classify $E$. coli strains is necessary.

Banerjee et al. reported ST131 was a dominant, antimicrobial-resistant clonal group associated with healthcare settings, elderly hosts, and persistent or recurrent symptoms [32]. Our findings show a stable existence of ST131 in a medical center in Taiwan. However, the prevalence of ST131 in patients with recurrent UTI was 17.2\% (unpublished data), higher than the prevalence of this clone in UTI patients' first episode in this study. We found 29 (87.9\%) and 5 (83.3\%) of phylogenetic group C isolates in 2009-2010 and 2020, respectively, were MDR-E. coli (Table 3). In contrast, only $140(44.7 \%)$ and $118(50.44 \%)$ B2 isolates in 2009-2010 and 2020, respectively, were MDR-E. coli (Table 3).

Recently, Chakraborty et al. collected $33 b l a_{\mathrm{NDM}-5}-$ producing $E$. coli isolates which were highly resistant to $\beta$-lactams, including novel $\beta$-lactam $/ \beta$-lactamase inhibitor combinations (ceftazidime/avibactam, imipenem/relebactam, and meropenem/vaborbactam). They found these isolates were assigned to different sequence types (STs) and indicated a predominance of isolates exhibiting ST167 in Switzerland and Germany $(n=10)$ (phylogenetic group C), followed by ST405 (group E), ST1284 (group C), and ST361 (group C) [33]. These results suggest that the new assigned phylogenetic groups, $C$ and E, may be responsible for the spread of MDR-E. coli worldwide. Therefore, the characteristics of E. coli phylogenetic groups $C$ and $E$ are worth investigating and comparing with other groups.

We found E. coli isolated in 2020 were, in general, more susceptible to many antimicrobials. These results suggest the decrease of antimicrobial resistance in 2020 may result from the implementation of the national antimicrobial stewardship program from 2013 in Taiwan. In addition, we showed that $E$. coli strains isolated from age group $>80$ were more resistant to most commonly used antimicrobial agents than strains isolated from age group $\leq 3$ (Table 4). These results are consistent with our previous findings [5]. In addition, Pulcini et al. also revealed that the elderly people in nursing homes had a risk around $40 \%$ higher than their community-dwelling peers of having antibiotic-resistant Enterobacteriaceae cultured from their urine samples [34]. Moreover, E. coli showed resistance to amoxicillin/clavulanate, nalidixic acid, ofloxacin, ciprofloxacin, ceftriaxone, and ESBLs were all more prevalent in nursing home samples than in community samples [34]. This observation may be caused by the high frequency and long-term use of antimicrobials in the elderly compared to the younger generation. As a result, antimicrobial stewardship and infection prevention and control programs should be tightly implemented in the elderly. 
Consistent with previous reports $[35,36]$, we found the high frequency of type 1 fimbrial adhesin gene fimH $(94.0 \%)$ in our 844 isolates (Table 5). These results suggest a critical role of FimH in establishing successful colonization of E. coli in the urinary tract. Our results showed a change in the prevalence of virulence factors in E. coli isolates in 2020 compared to 2009-2010 (Table 5). The significance of this evolutionary tract in E. coli uropathogenesis remains to be studied. In addition, the effect of different combinations of virulence factors in urinary tract colonization of E. coli is worth investigating. Here, our results revealed a decrease of virulence factors in E. coli isolated from the elderly compared to the younger generation (Table 6), which were consistent with our previous report, which showed the prevalence of virulence factors and antibiotic resistance of $E$. coli causing UTI were associated with patient age [5]. E. coli isolated from the elderly were more resistant to antimicrobials and had fewer virulence factors [5]. The role(s) of specific virulence factors in E. coli causing UTI in different patient age groups remains to be studied experimentally. Moreover, direct evidence demonstrating that the elderly are more vulnerable to low virulent $E$. coli caused by the decline of host immune protection or increase in host risk factors is still lacking.

In this study, we compared the characteristics of E. coli isolated from UTI patients in a single medical center at a 10-year interval to determine the regional evolutionary change of E. coli. We found a decrease in antimicrobial resistance and a difference in phylogenetic group composition and virulence factor distribution of E. coli at a 10-year interval. However, the driving force behind these phenotypic and genotypic changes among E. coli causing UTI at a 10-year interval remains to be investigated. In addition, whether these changes are associated with the virulence of E. coli is unclear. Moreover, our results showed that the characteristics of $E$. coli isolated from UTI patients were strongly associated with patient age. E. coli isolated from the elderly were more resistant to antimicrobials and had fewer virulence factors. Accordingly, the distinct characteristics of $E$. coli isolated from different age groups revealed in this study may be beneficial for clinical physicians to precisely control E. coli-caused UTI in different age groups of patients in the future.

\section{Materials and Methods}

\subsection{Sampling and Isolation of E. coli}

E. coli isolates were recovered from patients with UTIs at National Cheng Kung University hospital during 2009-2010 and 2020. This study was approved by the NCKUH Research Ethics Committee (B-ER-110-144). E. coli isolates were identified in the clinical laboratory by colony morphology, Gram stain, biochemical tests, and the Vitek system (bioMérieux, Marcy l'Etoile, France) according to the manufacturer's recommendations. A total of 504 and 340 non-duplicate E. coli isolates were collected in 2009-2010 and 2020, respectively. E. coli isolates were stored at $-80^{\circ} \mathrm{C}$ in lysogeny broth (LB) containing $20 \%$ glycerol $(v / v)$ until tested.

\subsection{Virulence Factors Identification}

$\mathrm{K} 1$ capsule antigen gene and 15 virulence factor genes of $E$. coli were detected by PCR according to previous studies $[5,25,37]$. Primer pairs specific for the K1 capsule gene (neuA), 3 PapG adhesion genes of P-fimbriae (papG class I to III), type 1 fimbrial adhesins $(f i m H)$, S-/F1C-fimbriae (sfa/foc), afimbrial adhesins (afa), iron-regulated gene homologue adhesin (iha), hemolysin (hlyA), cytotoxic necrotizing factor 1 (cnf1), catecholate siderophore receptor (iroN), ferric aerobactin receptor (iut $A)$, outer membrane protease $\mathrm{T}$ (ompT), and uropathogenic specific protein $(u s p)$, are listed in Table S3. Positive and negative control strains for the traits of interest were included in each assay.

\subsection{Phylogenetic Grouping and Escherichia coli Sequence Type 131 (ST131) Detection}

Based on PCR amplification patterns of specific genetic markers $(\operatorname{arp} A, \operatorname{chu} A, \operatorname{trp} A$, yjaA, and TSPE4.C2), E. coli strains were divided into eight phylogenetic groups: A, B1, 
B2, C, D, E, F, and clade I, according to the previous study [27]. The primers used for phylogenetic typing are listed in Table S3.

Primers ST131_for (5'-GACTGCATTTCGTCGCCATA-3') and ST131_rev (5'-CCGGCG GCATCATAATGAAA- $3^{\prime}$ ) in combined purified genomic DNA as a template were used to perform PCR to detect E. coli ST131, according to the previous study [38]. Amplification mixtures were performed with the following cycling conditions: an initial denaturation at $94{ }^{\circ} \mathrm{C}$ for $3 \mathrm{~min}, 30$ cycles of $94{ }^{\circ} \mathrm{C}$ for $30 \mathrm{~s}, 60^{\circ} \mathrm{C}$ for $30 \mathrm{~s}$, and $72{ }^{\circ} \mathrm{C}$ for $30 \mathrm{~s}$, and one final cycle of $72{ }^{\circ} \mathrm{C}$ for $5 \mathrm{~min}$.

\subsection{Antimicrobial Susceptibility Testing}

The antimicrobial susceptibility was experimentally determined through disk diffusion assay against 21 antibiotics, including amikacin $(30 \mu \mathrm{g})$, amoxicillin $(30 \mu \mathrm{g})$, ampicillin $(10 \mu \mathrm{g})$, ampicillin/sulbactam $(10 \mu \mathrm{g} / 10 \mu \mathrm{g})$, cefazolin $(30 \mu \mathrm{g})$, cefepime $(30 \mu \mathrm{g})$, cefmetazole $(30 \mu \mathrm{g})$, cefoxitin $(30 \mu \mathrm{g})$, ceftazidime $(30 \mu \mathrm{g})$, ceftriaxone $(30 \mu \mathrm{g})$, cefuroxime $(30 \mu \mathrm{g})$, ciprofloxacin $(5 \mu \mathrm{g})$, ertapenem $(10 \mu \mathrm{g})$, gentamicin $(10 \mu \mathrm{g})$, imipenem $(10 \mu \mathrm{g})$, meropenem $(10 \mu \mathrm{g})$, levofloxacin $(5 \mu \mathrm{g})$, piperacillin/tazobactam $(100 \mu \mathrm{g} / 10 \mu \mathrm{g})$, sulfamethoxazole/trimethoprim $(23.27 \mu \mathrm{g} / 1.25 \mu \mathrm{g})$, tetracycline $(30 \mu \mathrm{g})$, and tigecycline $(15 \mu \mathrm{g})$ (BD BBL Sensi-Disc, Becton, Dickinson and Company, Sparks, MD, USA) following the Clinical and Laboratory Standards Institute (CLSI) guidelines [39]. E. coli ATCC 25922 was used as our quality control strain. The interpretation of resistance to these antimicrobial agents was determined according to the recommendations of the CLSI [39].

\subsection{Statistical Analysis}

Pearson's Chi-square tests or Student $t$-tests were used for comparing categorical variables. All statistical analyses were performed using IBM SPSS statistics version 24.0 (IBM Corporation, Armonk, NY, USA). A $p$-value $<0.05$ was taken as significant.

Supplementary Materials: The following are available online at https:/ / www.mdpi.com/article/10 .3390 / pathogens10091156/s1, Table S1: The distribution of antimicrobial-resistant E. coli in different age groups in 2009-2010 and 2020, Table S2: The distribution of virulence factors in E. coli in different age groups in 2009-2010 and 2020, Table S3: Oligonucleotide primers used in this study.

Author Contributions: Data curation, W.-H.L.; Formal analysis, C.-Y.K.; Funding acquisition, C.-Y.K.; Investigation, Y.-Z.Z., P.-Y.L., P.-S.C., S.W., P.-Y.K., T.T.D.T., T.T.T.D., L.-L.W. and Y.-H.H.; Methodology, Y.-Z.Z.; Project administration, C.-Y.K.; Supervision, M.-C.W. and C.-Y.K.; Writing-original draft, C.-Y.K.; Writing-review \& editing, M.-C.W. and C.-Y.K. All authors have read and agreed to the published version of the manuscript.

Funding: This research was funded by the Ministry of Science and Technology (Taiwan), grant numbers 109-2320-B-010-036-MY3 and 110-2320-B-A49A-524-; the En Chu Kong Hospital (Taiwan), grant number ECKH_W11001; the Yen Tjing Ling Medical Foundation (Taiwan), grant number CI-110-13; and the National Yang-Ming University-Far Eastern Memorial Hospital Joint Research Program (Taiwan), grant number 110DN14.

Institutional Review Board Statement: Ethical review and approval were waived for this study, due to the use of these clinically obtained isolates were remnants from patient samples, and the data were analyzed anonymously.

Informed Consent Statement: Not applicable.

Conflicts of Interest: The authors declare no conflict of interest.

\section{References}

1. Medina, M.; Castillo-Pino, E. An introduction to the epidemiology and burden of urinary tract infections. Ther. Adv. Urol. 2019, 11, 1756287219832172. [CrossRef]

2. Geerlings, S.E. Clinical Presentations and Epidemiology of Urinary Tract Infections. Microbiol. Spectr. 2016, 4. [CrossRef]

3. Dubbs, S.B.; Sommerkamp, S.K. Evaluation and Management of Urinary Tract Infection in the Emergency Department. Emerg. Med. Clin. N. Am. 2019, 37, 707-723. [CrossRef] [PubMed]

4. Stapleton, A.E. Urinary tract infection pathogenesis: Host factors. Infect. Dis. Clin. N. Am. 2014, 28, 149-159. [CrossRef] [PubMed] 
5. Lin, W.H.; Wang, M.C.; Liu, P.Y.; Chen, P.S.; Wen, L.L.; Teng, C.H.; Kao, C.Y. Escherichia coli urinary tract infections: Host age-related differences in bacterial virulence factors and antimicrobial susceptibility. J. Microbiol. Immunol. Infect. 2021, in press. [CrossRef] [PubMed]

6. Reitzer, L.; Zimmern, P. Rapid Growth and Metabolism of Uropathogenic Escherichia coli in Relation to Urine Composition. Clin Microbiol. Rev. 2019, 33, e00101-19. [CrossRef] [PubMed]

7. Lee, J.H.; Subhadra, B.; Son, Y.J.; Kim, D.H.; Park, H.S.; Kim, J.M.; Koo, S.H.; Oh, M.H.; Kim, H.J.; Choi, C.H. Phylogenetic group distributions, virulence factors and antimicrobial resistance properties of uropathogenic Escherichia coli strains isolated from patients with urinary tract infections in South Korea. Lett. Appl. Microbiol. 2016, 62, 84-90. [CrossRef] [PubMed]

8. Tseng, C.C.; Lin, W.H.; Wu, A.B.; Wang, M.C.; Teng, C.H.; Wu, J.J. Escherichia coli FimH adhesins act synergistically with PapGII adhesins for enhancing establishment and maintenance of kidney infection. J. Microbiol. Immunol. Infect. 2020, in press. [CrossRef] [PubMed]

9. Tseng, C.C.; Wang, M.C.; Lin, W.H.; Liao, I.C.; Chen, W.C.; Teng, C.H.; Yan, J.J.; Wu, A.B.; Wu, J.J. Role of class II P fimbriae and cytokine response in the pathogenesis of Escherichia coli kidney infection in diabetic mice. J. Microbiol. Immunol. Infect. 2018, 51, 492-499. [CrossRef] [PubMed]

10. Wang, M.C.; Tseng, C.C.; Chen, C.Y.; Wu, J.J.; Huang, J.J. The role of bacterial virulence and host factors in patients with Escherichia coli bacteremia who have acute cholangitis or upper urinary tract infection. Clin. Infect. Dis. 2002, 35, 1161-1166. [CrossRef]

11. Wang, M.C.; Tseng, C.C.; Wu, A.B.; Lin, W.H.; Teng, C.H.; Yan, J.J.; Wu, J.J. Bacterial characteristics and glycemic control in diabetic patients with Escherichia coli urinary tract infection. J. Microbiol. Immunol. Infect. 2013, 46, 24-29. [CrossRef]

12. Luna-Pineda, V.M.; Moreno-Fierros, L.; Cazares-Dominguez, V.; Ilhuicatzi-Alvarado, D.; Ochoa, S.A.; Cruz-Cordova, A.; Valencia-Mayoral, P.; Rodriguez-Leviz, A.; Xicohtencatl-Cortes, J. Curli of Uropathogenic Escherichia coli Enhance Urinary Tract Colonization as a Fitness Factor. Front. Microbiol. 2019, 10, 2063. [CrossRef]

13. Garcia, E.C.; Brumbaugh, A.R.; Mobley, H.L. Redundancy and specificity of Escherichia coli iron acquisition systems during urinary tract infection. Infect. Immun. 2011, 79, 1225-1235. [CrossRef] [PubMed]

14. Johnson, J.R.; Jelacic, S.; Schoening, L.M.; Clabots, C.; Shaikh, N.; Mobley, H.L.; Tarr, P.I. The IrgA homologue adhesin Iha is an Escherichia coli virulence factor in murine urinary tract infection. Infect. Immun. 2005, 73, 965-971. [CrossRef] [PubMed]

15. Torres, A.G.; Cieza, R.J.; Rojas-Lopez, M.; Blumentritt, C.A.; Souza, C.S.; Johnston, R.K.; Strockbine, N.; Kaper, J.B.; Sbrana, E.; Popov, V.L. In vivo bioluminescence imaging of Escherichia coli O104:H4 and role of aerobactin during colonization of a mouse model of infection. BMC Microbiol. 2012, 12, 112. [CrossRef] [PubMed]

16. Garcia, T.A.; Ventura, C.L.; Smith, M.A.; Merrell, D.S.; O'Brien, A.D. Cytotoxic necrotizing factor 1 and hemolysin from uropathogenic Escherichia coli elicit different host responses in the murine bladder. Infect. Immun. 2013, 81, 99-109. [CrossRef] [PubMed]

17. Yamamoto, S.; Nakano, M.; Terai, A.; Yuri, K.; Nakata, K.; Nair, G.B.; Kurazono, H.; Ogawa, O. The presence of the virulence island containing the usp gene in uropathogenic Escherichia coli is associated with urinary tract infection in an experimental mouse model. J. Urol. 2001, 165, 1347-1351. [CrossRef]

18. He, X.L.; Wang, Q.; Peng, L.; Qu, Y.R.; Puthiyakunnon, S.; Liu, X.L.; Hui, C.Y.; Boddu, S.; Cao, H.; Huang, S.H. Role of uropathogenic Escherichia coli outer membrane protein $\mathrm{T}$ in pathogenesis of urinary tract infection. Pathog. Dis. 2015, 73 [CrossRef]

19. Johnson, J.R.; O’Bryan, T.T.; Delavari, P.; Kuskowski, M.; Stapleton, A.; Carlino, U.; Russo, T.A. Clonal relationships and extended virulence genotypes among Escherichia coli isolates from women with a first or recurrent episode of cystitis. J. Infect. Dis. 2001, 183, 1508-1517. [CrossRef]

20. Johnson, J.R.; Porter, S.; Johnston, B.; Kuskowski, M.A.; Spurbeck, R.R.; Mobley, H.L.; Williamson, D.A. Host Characteristics and Bacterial Traits Predict Experimental Virulence for Escherichia coli Bloodstream Isolates From Patients With Urosepsis. Open Forum. Infect. Dis 2015, 2, ofv083. [CrossRef]

21. Luo, Y.; Ma, Y.; Zhao, Q.; Wang, L.; Guo, L.; Ye, L.; Zhang, Y.; Yang, J. Similarity and divergence of phylogenies, antimicrobial susceptibilities, and virulence factor profiles of Escherichia coli isolates causing recurrent urinary tract infections that persist or result from reinfection. J. Clin. Microbiol. 2012, 50, 4002-4007. [CrossRef] [PubMed]

22. Bedenic, B.; Mestrovic, T. Mechanisms of Resistance in Gram-Negative Urinary Pathogens: From Country-Specific Molecular Insights to Global Clinical Relevance. Diagnostics 2021, 11, 800. [CrossRef] [PubMed]

23. Abd El Ghany, M.; Sharaf, H.; Al-Agamy, M.H.; Shibl, A.; Hill-Cawthorne, G.A.; Hong, P.Y. Genomic characterization of NDM-1 and 5, and OXA-181 carbapenemases in uropathogenic Escherichia coli isolates from Riyadh, Saudi Arabia. PLoS ONE 2018, 13, e0201613. [CrossRef] [PubMed]

24. Gurung, S.; Kafle, S.; Dhungel, B.; Adhikari, N.; Thapa Shrestha, U.; Adhikari, B.; Banjara, M.R.; Rijal, K.R.; Ghimire, P. Detection of OXA-48 Gene in Carbapenem-Resistant Escherichia coli and Klebsiella pneumoniae from Urine Samples. Infect. Drug Resist. 2020, 13, 2311-2321. [CrossRef] [PubMed]

25. Kao, C.Y.; Lin, W.H.; Tseng, C.C.; Wu, A.B.; Wang, M.C.; Wu, J.J. The complex interplay among bacterial motility and virulence factors in different Escherichia coli infections. Eur. J. Clin. Microbiol. Infect. Dis. 2014, 33, 2157-2162. [CrossRef] [PubMed]

26. Tseng, C.C.; Wu, J.J.; Liu, H.L.; Sung, J.M.; Huang, J.J. Roles of host and bacterial virulence factors in the development of upper urinary tract infection caused by Escherichia coli. Am. J. Kidney Dis. 2002, 39, 744-752. [CrossRef] 
27. Clermont, O.; Christenson, J.K.; Denamur, E.; Gordon, D.M. The Clermont Escherichia coli phylo-typing method revisited: Improvement of specificity and detection of new phylo-groups. Environ. Microbiol. Rep. 2013, 5, 58-65. [CrossRef]

28. Magiorakos, A.P.; Srinivasan, A.; Carey, R.B.; Carmeli, Y.; Falagas, M.E.; Giske, C.G.; Harbarth, S.; Hindler, J.F.; Kahlmeter, G.; Olsson-Liljequist, B.; et al. Multidrug-resistant, extensively drug-resistant and pandrug-resistant bacteria: An international expert proposal for interim standard definitions for acquired resistance. Clin. Microbiol. Infect. 2012, 18, 268-281. [CrossRef]

29. Iranpour, D.; Hassanpour, M.; Ansari, H.; Tajbakhsh, S.; Khamisipour, G.; Najafi, A. Phylogenetic groups of Escherichia coli strains from patients with urinary tract infection in Iran based on the new Clermont phylotyping method. Biomed Res. Int. 2015, 2015, 846219. [CrossRef]

30. Clermont, O.; Bonacorsi, S.; Bingen, E. Rapid and simple determination of the Escherichia coli phylogenetic group. Appl. Environ. Microbiol. 2000, 66, 4555-4558. [CrossRef]

31. Gordon, D.M.; Clermont, O.; Tolley, H.; Denamur, E. Assigning Escherichia coli strains to phylogenetic groups: Multi-locus sequence typing versus the PCR triplex method. Environ. Microbiol. 2008, 10, 2484-2496. [CrossRef]

32. Banerjee, R.; Johnston, B.; Lohse, C.; Porter, S.B.; Clabots, C.; Johnson, J.R. Escherichia coli sequence type 131 is a dominant, antimicrobial-resistant clonal group associated with healthcare and elderly hosts. Infect. Control. Hosp. Epidemiol. 2013, 34, 361-369. [CrossRef]

33. Chakraborty, T.; Sadek, M.; Yao, Y.; Imirzalioglu, C.; Stephan, R.; Poirel, L.; Nordmann, P. Cross-Border Emergence of Escherichia coli Producing the Carbapenemase NDM-5 in Switzerland and Germany. J. Clin. Microbiol. 2021, 59. [CrossRef]

34. Pulcini, C.; Clerc-Urmes, I.; Attinsounon, C.A.; Fougnot, S.; Thilly, N. Antibiotic resistance of Enterobacteriaceae causing urinary tract infections in elderly patients living in the community and in the nursing home: A retrospective observational study. $J$. Antimicrob. Chemother. 2019, 74, 775-781. [CrossRef]

35. Dadi, B.R.; Abebe, T.; Zhang, L.; Mihret, A.; Abebe, W.; Amogne, W. Distribution of virulence genes and phylogenetics of uropathogenic Escherichia coli among urinary tract infection patients in Addis Ababa, Ethiopia. BMC Infect. Dis. 2020, 20, 108. [CrossRef]

36. Pootong, A.; Mungkornkeaw, N.; Norrapong, B.; Cowawintaweewat, S. Phylogenetic background, drug susceptibility and virulence factors of uropathogenic E. coli isolate in a tertiary university hospital in central Thailand. Trop. Biomed. 2018, 35, 195-204.

37. Lin, W.H.; Tseng, C.C.; Wu, A.B.; Chang, Y.T.; Kuo, T.H.; Chao, J.Y.; Wang, M.C.; Wu, J.J. Clinical and microbiological characteristics of peritoneal dialysis-related peritonitis caused by Escherichia coli in southern Taiwan. Eur. J. Clin. Microbiol. Infect. Dis. 2018, 37, 1699-1707. [CrossRef]

38. Doumith, M.; Day, M.; Ciesielczuk, H.; Hope, R.; Underwood, A.; Reynolds, R.; Wain, J.; Livermore, D.M.; Woodford, N. Rapid identification of major Escherichia coli sequence types causing urinary tract and bloodstream infections. J. Clin. Microbiol. 2015, 53, 160-166. [CrossRef]

39. Clinical and Laboratory Standards Institute. Performance standards for antimicrobial susceptibility testing. In CLSI Supplement M100, 31st ed.; Clinical and Laboratory Standards Institute: Wayne, PA, USA, 2021. 